\title{
Online Application and Fast Solving Method for Critical Clearing Time of Three-Phase Short Circuit in Power System
}

\author{
Ling-Yu Ren*, Fang Tian, Jian-Feng Yan, Zhi-Hong Yu, Feng Su, Tao Wu \\ China Electric Power Research Institute, Beijing, 100192,China
}

\begin{abstract}
For transient stability analysis, the direct method runs fast but with poor accuracy due to the over-simplification of component models; the time-domain simulation method is accurate but time-consuming and thus not suitable for online application. In this paper, results of the Potential Energy Boundary Surface (PEBS) method applied on the simulation examples and actual system calculation are analyzed, and the range of error is discussed. Via complementarity of direct method and time-domain simulation method, a new hybrid method is proposed: in the calculation of the critical clearing time (CCT), the result of the direct method is used as the initial value and then the time-domain simulation method is employed for further search, which can meet the requirements of online application in terms of accuracy and speed. Based on the power system online dynamic security assessment and early warning system (the PDSA), the fast solving method of three-phase short-circuit CCT is applied on the East China $500 \mathrm{kV}$ power grid, and a detailed analysis of the result is presented. The engineering practice testifies the enhancement of CCT calculation in speed and accuracy.
\end{abstract}

Keywords: PEBS, time-domain simulation, hybrid method, dichotomy search, critical clearing time, online application

\section{Introduction}

With the wide access of renewable energy, power system is becoming increasingly complicated, and thus the calculation of transient stability limits is more significant. Currently, the two main tools for transient stability analysis (TSA) are the direct method and the time-domain simulation method. Based on strict mathematic models and algorithms, the time-domain simulation method can present exactly the operation of the power system under big disturbance (such as three-phase short circuit fault). The direct method can avoid the step-by-step (SBS) integration and generate a quantitative index, and thus is widely used in TSA. The recent study on the direct method mainly focuses on the transient energy function (TEF) method, and the research directions include: 1) modification of the algorithm itself [1]-[4]; 2) combination with other algorithm [5],[6]; 3) engineering project application [7]-[9].

The critical clearing time (CCT) of three-phase short circuit is an important parameter which shows the transient stability limit of power system. The research on CCT calculation by the direct method concentrates on improvement of the accuracy [10],[11]; the corresponding study on CCT solved by the time-domain simulation method focuses on enhancement of the speed[12]-[14]. The method proposed in [15] combines the advantages of the two methods, where the direct method is used for fault scanning and the time-domain simulation method is used for those serious faults. However, this method does not consider the possible omission of serious faults caused by the inaccuracy of the direct method and does not directly shorten the time for CCT calculation.

On the basis of the previous work, this paper proposes a hybrid method and realizes its online

* Manuscript received June 2, 2012; revised July 6, 2012.

Corresponding author. Tel.: +86-15201277089; E-mail address: renlingyu@epri.sgcc.com.cn. 
application on PDSA (the dynamic security assessment\& early warning system of power system) [16]. In this paper, the error range of the PEBS (potential energy boundary surface) method is discussed, then the results of PEBS method are used to provide search ranges of further CCT calculation by the time-domain simulation method, and finally this new method is testified by the simulation on $500 \mathrm{kV}$ power grid of East China.

\section{Time-Domain Simulation Method}

Based on the detailed models and the SBS integration algorithm, the time-domain simulation method can reflect all the complex dynamic features of power system under various disturbances. Firstly, this method establishes the differential-algebraic equation (DAE) (1) of power system. The mathematic models include description of the primary grid (the algebraic equations), the dynamic characteristics of the generators, loads, reactive compensation, HVDC (High Voltage Direct Current) transmission systems, etc. (the differential equations), and the possible disturbance manners, the control measures, etc. Then the DAE is solved with a certain steady-state power flow as its initial solution.

$$
\left\{\begin{array}{l}
\dot{\mathbf{X}}=\mathbf{F}(\mathbf{X}, \mathbf{Y}) \\
\mathbf{Y}=\mathbf{G}(\mathbf{X}, \mathbf{Y})
\end{array}\right.
$$

where $\mathbf{F}=\left(f_{1}, f_{2}, \ldots, f_{n}\right)^{T}, \mathbf{G}=\left(g_{1}, g_{2}, \ldots, g_{n}\right)^{T}$; and $\mathbf{X}=\left(x_{1}, x_{2}, \ldots, x_{n}\right)^{T}$ is the variables of differential equations; $\mathbf{Y}=\left(y_{1}, y_{2}, \ldots, y_{n}\right)^{T}$ is the variables of algebraic equations.

In this paper, the trapezoidal implicit integration method is adopted in the iterative algorithm of differential equations; the direct triangular decomposition method is combined with the iterative algorithm to solve the algebraic equations; the iterations of the differential equations and the algebraic equations run alternately until they converge and this is the solving process of one time step. Under the condition of faults, the differential equations are not continuous when the occurrence and remove of the faults lead to changes of the admittance matrix. At this time, the DAE needs to be modified and solved separately in different time intervals.

Currently, the time-domain simulation method is very mature in terms of mathematic model, calculation algorithm and programming, and as a powerful tool for offline analysis it has been broadly used in practical projects. The major advantages of this method include: 1) precise models; 2) good numerical stability; 3) ability to test the validity of other analytical methods for various physical problems and control strategies. With the enlargement of the power grid, the requirement for real-time analysis is upgraded and thus the weaknesses of the time-domain simulation method are becoming obvious: 1) huge workload and long work time for transient stability analysis of large-scale power system; 2) inability to provide a quantitative stability index; 3) low utilization of the massive information achieved in the simulation process.

The CCT calculation by time-domain simulation usually adopts the dichotomy method and considers the point at CCT as the not-return-point (NRP). The detailed process is as follows: firstly, set the initial clearing time, $t_{0}=0 \mathrm{~s}, t_{e}=5 \mathrm{~s}$ ( The initial search range is set to be $0-5 \mathrm{~s}$ for the reason that CCT larger than 5 seconds will not influence the system stability due to the action of relay and regulation devices.); then determine the system stability at the middle clearing time $t_{m}=\left(t_{0}+t_{e}\right) / 2$. The transient stability criterion is the traditional rotor angle criterion and the maximum rotor angle is set to be 500 degrees. If the system is stable, then reset the start time $t_{0}=t_{m}$; otherwise, reset the end time $t_{e}=t_{m}$. Lastly, cycle the process until $t_{0}-t_{e}$ reaches the designated precision scope. Since the initial search range is set conservatively, the number of cycles is very large in this method and the calculation speed is low for analysis of large-scale power system. 


\section{PEBS Method}

The PEBS method is one of the TEF methods which analyze the stability problem with an energy view. This method assumes that the potential energy changes slowly near the unstable equilibrium point (UEP) and thus the intersection of the continuous fault track and the PEBS can be used to approximate the UEP. This method need not calculate the related unstable equilibrium point (RUEP), and can incorporate the complicated device models after properly establishing the energy function.

The detailed process of CCT calculation by the PEBS method is illustrated in Fig.1. ( $V_{p}$ means the potential energy; $V_{c r}$ means the critical energy.)

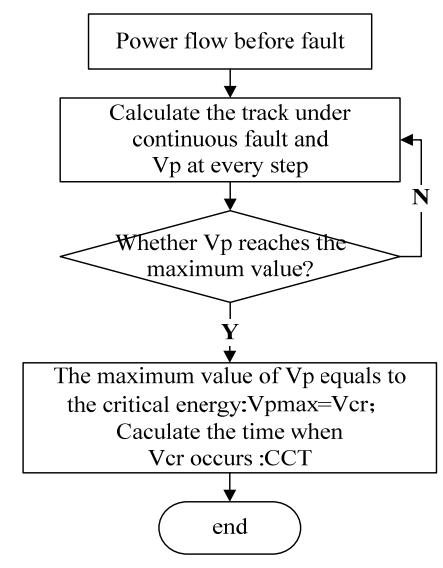

Fig. 1. Flow chart of CCT calculation by PEBS method

The main advantages of the TEF method are: 1) fast speed; 2) ability to provide an energy criterion for stability assessment and give stability margin. Although the TEF method trends to be more mature, there are many shortages and limits in practical application, which include: 1) poor adaptability to models; 2) inability to provide time-domain responses of system variables; 3 ) inability to point out the reason why a system is unstable or how it becomes unstable; 4) inability to reflect stability of system with switching operation. So far, the TEF method is still in development and more often it is used as an important complement of the time-domain simulation method.

\section{Error Analysis of the PEBS Method}

In the PEBS method, the conservation property of transient energy counts on the precision of the energy expression. The calculation of $V_{p}$ needs the constant impedance model and the classic generator model, and this simplification of models will bring error. To study this error, the New England 10 machine example is scanned and the CCT at every possible bus is calculated. The result is shown in Table 1.

Table 1. Error analysis of CCT calculation by the PEBS method on New England example

\begin{tabular}{l|lllllll}
\hline Fault bus number & 29 & 38 & 26 & 28 & 16 & 27 & 34 \\
\hline PEBS method & 0.1 & 0.1 & 0.11 & 0.11 & 0.15 & 0.15 & 0.15 \\
TDS method & 0.103 & 0.093 & 0.122 & 0.122 & 0.122 & 0.151 & 0.142 \\
Error & $-2.9 \%$ & $7.5 \%$ & $-9.8 \%$ & $-9.8 \%$ & $9.4 \%$ & $-0.66 \%$ & $5.6 \%$ \\
\hline
\end{tabular}

In this table, TDS method means the time-domain simulation method whose results are used as the criteria of CCT calculation. Only the top seven buses with the least CCT is listed for the sake of brevity. From table 1, it is revealed that the error of PEBS method is acceptable and keeps below $10 \%$. In this paper, the one-fold redundancy is adopted and thus the error is set to be $20 \%$.

As to practical application, the fault scanning is carried out on the $500 \mathrm{kV}$ power grid of East China, and the statistic of the result is shown in Fig. 2 (absolute value). 


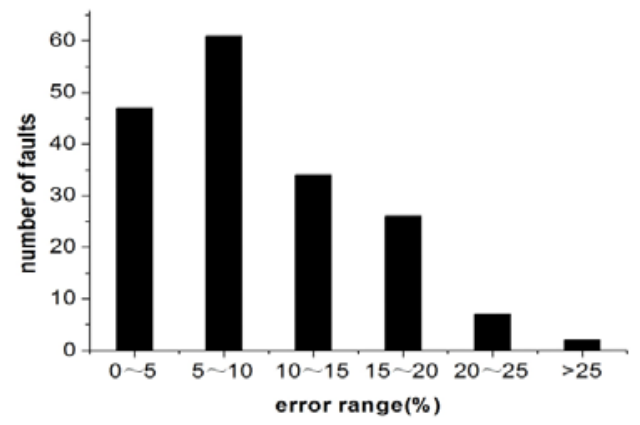

Fig. 2.Error distribution of CCT calculation by the PEBS method on East China grid

Contrast to the condition of the simple 10 machine example, in practical projects, the first task of CCT calculation by PEBS method is to simplify models and due to the complexity of modern power grid this simplification will conceal some important parameters. Thereby, there are some divergent points and points with oversize error where the further time-domain simulation will be in need for recalculation.

\section{Hybrid Method}

\subsection{Critical clearing time of three-phase short circuit fault}

The fault clearing time is the time between the occurrence of the fault and its complete abscission. The critical clearing time $t_{c r}$ is the maximum clearing time to keep the system stable, which meets the following conditions:

$$
\begin{cases}t<t_{c r} & \text { stable } \\ t=t_{c r} & \text { critical stable } \\ t>t_{c r} & \text { unstable }\end{cases}
$$

The three-phase fault is the most serious kind of fault, and its critical clearing time can reflect the transient stability of power system. Generally, the longer the CCT is, the stronger the system is in resisting the fault and the larger the corresponding stability margin.

The CCT can be calculated by the TEF method or the time-domain simulation method: the former concentrates on calculation of the critical energy or the critical rotor angle and the later needs to modify the clearing time several times to successively approximate the CCT.

\subsection{Hybrid method}

The hybrid method, as mentioned above, is actually the time-domain simulation method with the initial calculated by the PEBS method, whose detailed process is as follows:

1) Calculate CCT $t_{c r}$ by the PEBS method;

2) Set the initial value of the CCT range: $\min =t_{c r}(1-$ error $), \max =t_{c r}(1+$ error $)$;

3) Calculate the initial power flow;

4) Set the step of time-domain simulation: $D_{t}$;

5) Set the fault time, where the start time is: $T_{s}=0$, the end time is: $T_{e}=(\min +\max ) / 2$;

6) Carry out the transient stability calculation with the time-domain simulation method;

7) Search the minimum interval with the dichotomy method, $\max -\min <D_{t}$ ?;

8) Determine whether CCT is found, if not, reset the search range according to the result;

9) End.

In step 1), the parameter error is set to be $20 \%$ as has been discussed in chapter 4 . In step 2), the maximum value and the minimum value of CCT is defined as max, min. In step 7), the termination 
condition is set to be the fact that the integral interval max-min is less than the integral step $D_{t}$, which is consistent with that of the time-domain simulation. In step 8), whether the calculation succeeds is determined by the stability conditions at the two endpoints max, min: if the system is stable at both the two endpoints, then the calculation fails and the search range is reset to be $\min ^{\prime}=\max , \max ^{\prime}=5$ (the new value of max, $\min$ is defined as $\max ^{\prime}, \min ^{\prime}$ ); if the system is unstable at both the two endpoints, then the calculation fails and the search range is reset to be $\min ^{\prime}=0, \max ^{\prime}=\min$; if the system is stable at the point min and unstable at the point max , then calculation succeeds.

The flow chart of the CCT calculation by the hybrid method is shown in Fig.3.

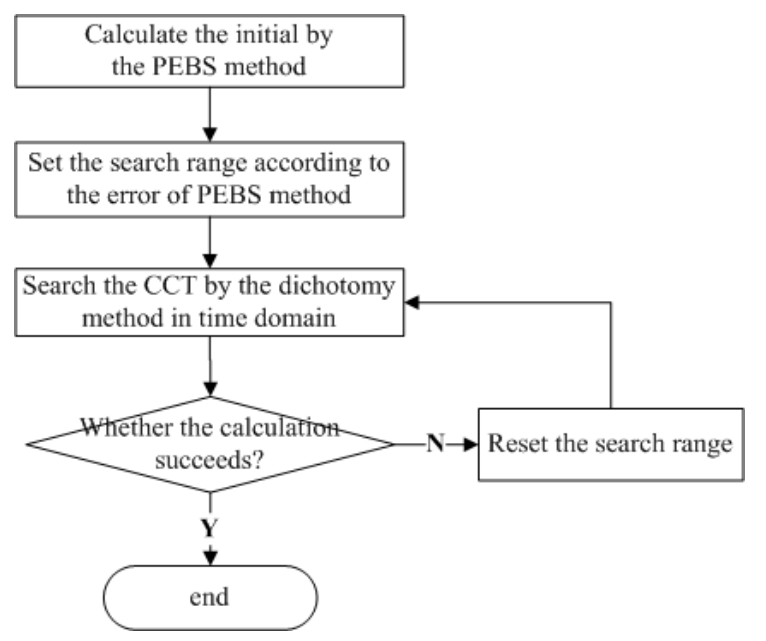

Fig. 3.Flow chart of CCT calculation by the hybrid method

\section{Online application}

The Power System Online Dynamic Security Assessment and Early Warning System (PDSA), which is developed by the China Electric Power Research Institute (CEPRI), is a set of mature software for power system stability analysis and has been employed by many province level dispatch centres in China. In this paper, the hybrid method for three-phase short circuit CCT calculation is integrated into the PDSA working platform and put into online application on the East China grid.

\subsection{System configuration of CCT calculation by hybrid method on PDSA}

1) Data integration

Data integration is an important function of PDSA dynamic data platform, which is in charge of the identification and examination of online data detection, the automatic integration of data in large-scale power grid and the automatic matching of online/offline data, so as to provide more comprehensive and accurate data of the entire grid for higher application.

2) Selection of fault set

According to the historical data and results from the offline analysis, the serious fault set, composed of faults at weak buses, will be selected for further study.

3) Fast solving of CCT

Based on the hybrid method proposed in this paper, the serious faults are further studied in order to obtain time margin index and determine whether it is necessary to activate the calculation of assistant decision.

4) Assistant decision

As to the faults that may cause system instability, the assistant decision can prevent the possible failure by adjusting the operating condition before fault to enhance the system's ability of disturbance resistance.

The system block diagram of CCT calculation by hybrid method on PDSA is shown in Fig.4. 


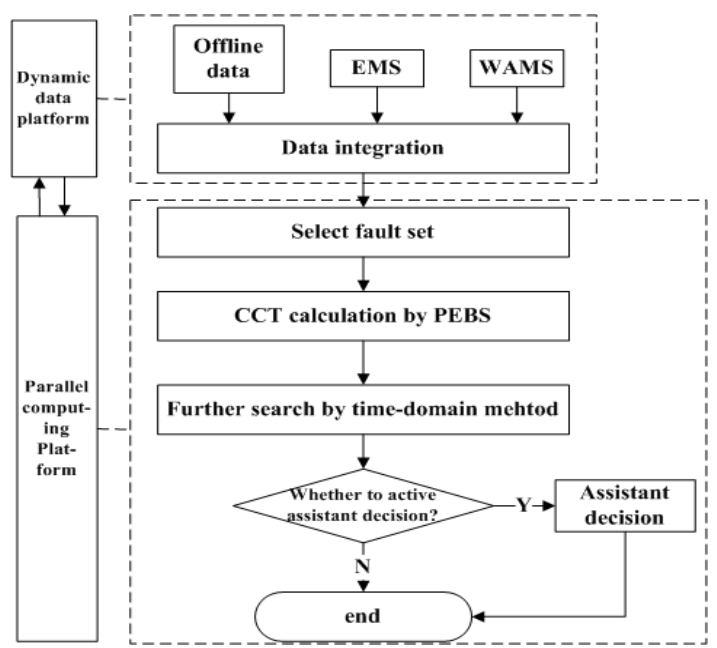

Fig. 4. System block diagram of CCT calculation by hybrid method on PDSA

Table 2. Result of CCT calculation on the $500 \mathrm{kV}$ power grid of East China

\begin{tabular}{lllll}
\hline Fault Number & Name of fault bus & PEBS method CCT(second) & Hybrid method CCT(second) & Error(\%) \\
\hline 36 & Zhefuyang & 0.11 & 0.12 & -8.33 \\
29 & Zheqiaosi & 0.12 & 0.13 & -7.7 \\
118 & Shipai & 0.12 & 0.12 & 0 \\
150 & Changshu & 0.13 & 0.11 & 18.2 \\
107 & Minputian & 0.14 & 0.17 & -17.6 \\
41 & Zheshuanglong & 0.15 & 0.20 & 25 \\
100 & Minfuzhou & 0.16 & 0.15 & 6.67 \\
103 & Mindayuan & 0.16 & 0.19 & -15.8 \\
102 & Minquanzhou & 0.17 & 0.18 & -5.56 \\
104 & Minjinjiang & 0.17 & 0.19 & -10.5 \\
\hline
\end{tabular}

\subsection{Simulation example and result analysis}

In this paper, the simulation is carried out on the $500 \mathrm{kV}$ power grid of East China which contains Jiangsu, Zhejiang, Fujian, Anhui and Shanghai grid, including 176 buses in 500kV level. After the fault set selection, a total number of 56 buses are scanned with the PEBS method, the time-domain simulation method and the hybrid method mentioned above, and the result is shown in Table 2.

This table lists the ten top buses with the least CCT and compares the accuracy of the PEBS method with that of the hybrid method. (Number of fault is the internal code in PDSA.) From this table, among the then buses, only bus Zheshuanglong has the PEBS result with an error over $20 \%$, that is to say only one bus needs a second search. Moreover, based on the principle of the hybrid method, thanks to the same convergence condition, the result of this new method has the same accuracy with the time-domain simulation method.

The calculation time is shown in Table 3:

Table 3. CCT calculation time on the $500 \mathrm{kV}$ power grid of East China

\begin{tabular}{llll}
\hline & Time-domain simulation & Hybrid method & Saving (\%) \\
\hline Computing time (minute) & 29.34 & 10.75 & 63.36 \\
\hline
\end{tabular}

This table compares the computing time of the time-domain method and the hybrid method on the $500 \mathrm{kV}$ power grid of East China. Since the new method reduces the search range, the number of circulation decreases and the simulation shows that the hybrid method can save nearly 2/3 of the computing time compared with traditional method. With the increase of the magnitude of studied power grid, this merit will be more evident. 


\section{Conclusion}

As a common tool for simple analysis of power system, the PEBS method is limited by the accuracy of its models and the statistic shows the error can basically keep below 20\%; the hybrid method mentioned in this paper can combine the merits of the PEBS method (fast speed) and the time-domain simulation method (high accuracy), and simulation results demonstrate that it is more competent in online application.

\section{References}

[1] Chen L, Min Y. Transient stability analysis of power systems by direct method considering transient voltage Dip. Automation of Electric Power Systems, 2006; 30(21):6-10.

[2] Xia C, Ru F, Hu H, et al. A novel transient energy margin computation method based on fault trajectory. Relay, 2004; 32(18):1-5.

[3] Bai XF, Jiang T, Guo ZZ. Dynamic integral step control of Taylor series in transient energy function method. Electric Power Automation Equipment, 2004; 24(3):29-31.

[4] Fang D, Zhang Y, Song W, et al. Corrected transient energy function and its application to power system transient stability assessment. Proceedings of the CSEE, 1998; 18(3):200-203.

[5] Liu H, Min Y, Zhang Y, et al. Credible region and its extension for approximate boundaries of transient stability region in power system. Proceedings of the CSEE, 2008; 28(31):9-14.

[6] Ma J, Cheng D, Mei S. Approximation on boundary of power system stability region based on semi-tensor theory: part one theoretical base. Automation of Electric Power Systems, 2006; 30(10):1-5.

[7] Lin Y, Cai Z. Transient stability analysis of AC/DC power system based on PEBS method. Electric Power Automation Equipment, 2009; 29(1):24-28.

[8] Yu T, Wei M, Chan KW. Application of transient energy function method in auxiliary decision of black start of local power grids. Automation of Electric Power Systems, 2006; 30(13):73-78.

[9] Chung TS, Fang DZ. A fast approach to transient stability estimation using an improved potential energy boundary surface method. Electric Power Systems Research, 1995; 34(1):47-55.

[10] Yan C, Zhou X. Online application of solving method for critical clearing time of three-phase short circuit in power system. Power System Technology, 2009; 33(17):56-63.

[11] Xue A, Mei S, Ni Y, et al. Critical clearing time estimation of power system based on quadratic approximation of stability region. Automation of Electric Power Systems, 2005; 29(19):19-24.

[12] Fang D, Qin Y. A new trajectory sensitivities approach for CCT assessment. Proceedings of the CSEE, 2005; 25(14):7-11.

[13] Xie D, Yan Z, Wang X, et al. Parallel transient stability assessment with adaptive multi-section methods. East China Electric Power, 2008; 36(12):16-20.

[14] Wang S, Zhang B, Liu Y. Critical clearing time interval calculation and its application in contingency screening. Power System Technology, 2003; 27(10):72-77.

[15] Chang H, Liu W, Xing Z, et al. Online application of power system transient stability computation. Power System Technology, 2007; 31(13):54-58.

[16] Yan J, Yu Z, Tian F, et al. Dynamic security assessment\& early warning system of power system. Proceedings of the CSEE, 2008; 28(34):87-93. 